\title{
Bayesian estimation of the half-normal regression model with deterministic frontier
}

FRANCISCO J. ORTEGA

Avenida Ramón y Cajal 1, Sevilla, Spain.

Universidad de Sevilla, Sevilla, Spain

Phone: +34954556970

e-mail: fjortega@us.es

JOSE M. GAVILAN

Avenida Ramón y Cajal 1, Sevilla, Spain

Universidad de Sevilla, Sevilla, Spain

Phone: +34 954556970

e-mail: gavi@us.es 


\title{
Bayesian estimation of the half-normal regression model with deterministic frontier
}

\begin{abstract}
A regression model with deterministic frontier is considered. This type of model has hardly been studied, partly owing to the difficulty in the application of maximum likelihood estimation since this is a non-regular model. As an alternative, the Bayesian methodology is proposed and analysed. Through the Gibbs algorithm, the inference of the parameters of the model and of the individual efficiencies are relatively straightforward. The results of the simulations indicate that the utilized method performs reasonably well.
\end{abstract}

Keywords Deterministic Frontier, Bayesian estimation, Gibbs algorithm.

JEL Classification C11, C15, D24. 


\section{INTRODUCTION}

The origin of the analysis of the econometric models with frontier and the calculation of efficiency measures can be set in Farrel (1957), where the innovative idea of analysing the efficiency of a productive process is established in terms of the observed deviations between the actual production and an ideal frontier of maximum output. Aigner and Chu (1968), following the initiative of Farrel (1957), propose a model where a given output is expressed as a function of a series of inputs and unknown parameters plus a negative random perturbation; the deterministic part of the model corresponds to the production frontier or maximum attainable value of output given the inputs, and the random perturbation (difference between the actual production and its potential maximum value) represents the level of inefficiency in the productive process. This formulation of the model is known as the deterministic frontier model (DFM)

After the appearance of the stochastic frontier models (SFM), formulated for the first time by Aigner et al. (1977), Battese and Corra (1977), and Meeusen and van den Broeck (1977), the interest of the researchers in the DFM quickly declined in favour of the SFM. In the latter, a composite error term is introduced in the form of two random perturbations. The first is an inefficiency measure and the other represents all the possible sources of random variations.

In the specific literature, there are a number of negative results concerning the DFM. It has been argued that this type of modelization does not take into account any source errors nor does it consider any other kind of random variations and therefore they are assigned to inefficiency in the productive process. Another drawback of this modelization is that it poses a non-regular problem, and therefore the properties of the maximum likelihood estimator (MLE) are uncertain. In Greene (1980), this latter disadvantage is partially solved: naming $\varepsilon$ the inefficiency, it is established that if the density of $\mathcal{E}$ and its derivative at the point $\varepsilon=0$ are equal to zero, then the DFM verifies the usual conditions of regularity. Several distributions, such as the log-normal distribution, and the gamma distribution with shape parameter greater than or equal to two, fulfil the aforementioned conditions, while other commonly used distributions, such as the exponential and the half-normal distributions, fail to fulfil the conditions.

Nor is the SFM free of drawbacks. In many cases, it is difficult to disentangle the random effect and the inefficiency of the composite error, and there is a significant proportion of samples that involve one or the other extreme (absence of inefficiency or absence of random errors), even for large sample sizes (Simar, 2007; Ortega and Gavilan, 2011). Therefore, consideration of the DFM (or at least the comparison of the result with the SFM) can be especially interesting when the estimation of the SFM suggests the absence of random errors 
and, as a consequence, that $100 \%$ of the composite error is due to inefficiency. As a matter of fact, the software to estimate the SFM in this situation usually provides some kind of warning or error message. To be specific, using the version 1.1-0 of the package frontier in the software $\mathrm{R}$ (Coelli and Henningsen, 2013) the following warning message is obtained ${ }^{1}$ :

the parameter 'gamma' is close to the boundary of the parameter space $[0,1]$ : this can cause convergence problems and can negatively affect the validity and reliability of statistical tests and might be caused by model misspecification

In the same way, version 9 of the software LIMDEP gives the following error message, stops the estimation, and no result is provided:

Error 143: Models - estimated variance matrix of estimates is singular

In Amsler et al. (2013), the DFM is analysed in a number of situations, concluding that in some simple cases it is possible to make an exact inference using analytic expressions for the MLE for finite samples. In models of a more complicated nature, the exact inference remains possible, although simulation processes are necessary to obtain the critical points. This property is interesting, since it cannot be forgotten that, in the SFM, the properties of the MLE and the inference process in general are asymptotic, and therefore imply limitations when the sample size is small or moderate.

In this paper, the Bayesian estimation of the DFM through the Gibbs algorithm (Gelfand and Smith, 1990) is presented as an alternative. The Bayesian approach has been widely developed and utilized in the SFM, particularly since the publication of the paper by Van de Broeck et al. (1994). In Griffin and Steel (2007), a number of formulations of the SFM and their implementation in the software of Bayesian estimation WinBUGS are presented. However, to the best of our knowledge, the Bayesian literature has paid little attention to the DFM.

The Bayesian approach to the DFM holds interesting advantages, such as the ease of adding constraints involving both the parameters and the observations, which make the model nonregular, or the possibility of obtaining exact inferences for finite samples, not only for the parameters of the model but also for the individual efficiencies (although simulation processes are required to obtain the estimated values).

The rest of the paper is organized as follows: in Section 2, the model, and the prior and the posterior distributions, are presented. In Section 3, the Gibbs algorithm for the considered model is developed. In Section 4, the properties of the estimators are analysed through

\footnotetext{
${ }^{1}$ The parameter $\gamma$ mentioned is the proportion of the total variance of the composite error due to inefficiency. Therefore, if it is estimated to be 1 , then the whole error is inefficiency, that is to say, the model does not have random noise and its frontier is deterministic.
} 
simulation, and in Section 5, two well-known examples are revisited. Finally, in Section 6, the main conclusions of the paper are discussed.

\section{THE MODEL: PRIOR AND POSTERIOR DISTRIBUTIONS}

Let us consider the following production model:

$$
y_{i}=\mathbf{x}_{\mathbf{i}}^{\prime} \boldsymbol{\beta}-\varepsilon_{i}, \quad i=1, \ldots, n
$$

where $y_{i}$ is the production, $\boldsymbol{\beta} \in \mathbb{R}^{k}$ is a vector of unknown parameters (hence, $k$ is the number of parameters to estimate in the production function), $\mathbf{x}_{\mathbf{i}}$ is the vector of exogenous variables (with $x_{i 1}=1 \forall i$, that is, the model has an intercept corresponding to the parameter $\beta_{1}$ ), n is the sample size, and $\varepsilon_{i}>0$ is a random perturbation that measures the inefficiency of the $i$-th observation. The hypothesis that $\varepsilon_{i}$ follows a half-normal distribution $\left(0, \sigma^{2}\right)$ is assumed (Johnson et al., 1994; Pewsey, 2004), that is, the probability density function of $\varepsilon_{i}$ is given by:

$$
f\left(\varepsilon_{i}\right)=\frac{2}{\sqrt{2 \pi \sigma^{2}}} \exp \left\{-\frac{\varepsilon_{i}^{2}}{2 \sigma^{2}}\right\}, \varepsilon_{i}>0
$$

and therefore, the probability density function of $y_{i}$ is:

$$
f\left(y_{i} \mid \mathbf{x}_{\mathbf{i}}, \boldsymbol{\beta}, \sigma\right)=\frac{2}{\sqrt{2 \pi \sigma^{2}}} \exp \left\{-\frac{1}{2 \sigma^{2}}\left(y_{i}-\mathbf{x}_{\mathrm{i}}^{\prime} \boldsymbol{\beta}\right)^{2}\right\}, y_{i} \leq \mathbf{x}_{\mathbf{i}}^{\prime} \boldsymbol{\beta}
$$

The likelihood function of the model for a sample of size $n$ is given by

$$
L(\boldsymbol{\beta}, \sigma \mid \mathbf{y}, \mathbf{X}) \propto\left(\sigma^{2}\right)^{-n / 2} \exp \left\{-\frac{1}{2 \sigma^{2}} \sum_{i}\left(y_{i}-\mathbf{x}_{\mathbf{i}}^{\prime} \boldsymbol{\beta}\right)^{2}\right\}, y_{i} \leq \mathbf{x}_{\mathbf{i}}^{\prime} \boldsymbol{\beta} \forall i=1, \ldots, n
$$

where $\mathbf{y}=\left(y_{1}, \ldots, y_{n}\right)^{\prime} \in \mathbb{R}^{n}$ and $\mathbf{X}^{\prime}=\left[\mathbf{x}_{1}|\ldots| \mathbf{x}_{\mathbf{n}}\right] \in \mathbf{M}_{k \times n}$.

In order to simplify the notation, the set $\mathrm{B}=\left\{\boldsymbol{\beta} \in \mathbb{R}^{k} / y_{i} \leq \mathbf{x}_{\mathrm{i}}^{\prime} \boldsymbol{\beta} \forall i=1, \ldots, n\right\}$ is defined, that is, $B$ is the parametric space or set of values of the parameter $\boldsymbol{\beta}$ that is compatible with the observed set of data according to the established model. As a consequence, the constraints can be expressed as $\boldsymbol{\beta} \in B$. The logical hypothesis that the set $B$ is not empty is also assumed.

Let us point out that the stochastic frontier production model is formulated as: 


$$
y_{i}=\mathbf{x}_{\mathbf{i}}^{\prime} \mathbf{\beta}-\varepsilon_{i}+u_{i}, \quad i=1, \ldots, n
$$

where the hypothesis of the model (1) holds and it is also assumed that $u_{i}$ follows a Normal distribution $\left(0, \sigma_{U}^{2}\right)$, and that there is independence between the perturbations $\varepsilon_{i}$ and $u_{i}$. In this setting, a number of values $y_{i}$ could be above $\mathbf{x}_{\mathbf{i}}^{\prime} \boldsymbol{\beta}$ due to the noise perturbation $u_{i}$. In this way, the constraints $y_{i} \leq \mathbf{x}_{\mathbf{i}}^{\prime} \boldsymbol{\beta}, \quad i=1, \ldots, n$ are removed, thus resulting in a likelihood without constraints.

As mentioned in Section 1, the interest is focused on the deterministic frontier model. The set of unknown parameters of the model (1) is $(\boldsymbol{\beta}, \sigma)$, where $\boldsymbol{\beta} \in B$ and $\sigma>0$. The Bayesian inference is based on the determination of the joint posterior distribution of the parametric vector $(\boldsymbol{\beta}, \sigma)$ given the set of data, such posterior distribution is denoted by $\pi(\boldsymbol{\beta}, \sigma \mid \mathbf{y}, \mathbf{X})$. In order to obtain the posterior distribution it is necessary, first of all, to choose a joint prior distribution for the vector of parameters $(\boldsymbol{\beta}, \sigma)$; such prior distribution is denoted by $\pi(\boldsymbol{\beta}, \sigma)$.

In the setting of the Bayesian analysis of the linear regression model, a normal prior distribution for $\boldsymbol{\beta}$ and inverse gamma for $\sigma^{2}$ are frequently chosen; this Gaussian-inverse gamma distribution has the advantage of being conjugate, that is to say, the posterior distribution is also Gaussian-inverse gamma, which facilitates the inference process. However, this distribution is informative, because the hyperparameters must be chosen based on prior knowledge of the problem.

In this paper, a non-informative prior distribution has been chosen, which eliminates the need for choosing the hyperparameters and represents the cases in which "little is known about the values of $\boldsymbol{\beta}$ and $\sigma^{2}$. A number of methods to obtain non-informative prior distributions have been proposed. In the linear regression model, the non-informative prior distribution most commonly used (Jeffreys, 1961, p.182; Box and Tiao, 1973, p.117) is:

$$
\pi(\boldsymbol{\beta}, \sigma) \propto \sigma^{-1}
$$

In Zellner (1986), an excellent review of the linear regression model with various prior distributions is offered.

The kernel of the joint posterior distribution is calculated by multiplying the likelihood function (4) by the prior distribution (6), thereby obtaining:

$$
\pi(\boldsymbol{\beta}, \sigma \mid \mathbf{y}, \mathbf{X}) \propto\left(\sigma^{2}\right)^{-(n+1) / 2} \exp \left\{-\frac{1}{2 \sigma^{2}} \sum_{i}\left(y_{i}-\mathbf{x}_{\mathbf{i}}^{\prime} \boldsymbol{\beta}\right)^{2}\right\}, \boldsymbol{\beta} \in \mathrm{B}
$$


which can be expressed as

$$
\pi(\boldsymbol{\beta}, \sigma \mid \mathbf{y}, \mathbf{X}) \propto\left(\sigma^{2}\right)^{-(n+1) / 2} \exp \left\{-\frac{1}{2 \sigma^{2}}(\mathbf{y}-\mathbf{X} \boldsymbol{\beta})^{\prime}(\mathbf{y}-\mathbf{X} \boldsymbol{\beta})\right\}, \boldsymbol{\beta} \in \mathrm{B}
$$

As may be seen, the use of this non-informative prior distribution also leads to a Gaussianinverse gamma kernel for the posterior distribution. However, the algebraic manipulation of this distribution is not easy, since the constraint $\boldsymbol{\beta} \in B$ makes it difficult to ascertain the integration limits, and forces the marginal distribution of each component $\beta_{j}$ to be defined piecewise. In order to avoid this complication and to give an answer to the inference problem, the Gibbs algorithm is utilized (Gelfand and Smith, 1990).

\section{FORMULATION OF THE GIBBS ALGORITHM}

As pointed out in Section 2, the kernel of the posterior distribution is Gaussian-inverse gamma. Therefore, $\sigma^{-2} \mid \boldsymbol{\beta}, \mathbf{y}, \mathbf{X}$ follows a gamma distribution and $\boldsymbol{\beta} \mid \sigma, \mathbf{X}, \mathbf{y}$ follows a normal kdimensional distribution (in this case truncated to the constraint $\boldsymbol{\beta} \in B$ ).

Indeed, by taking into consideration that the joint posterior distribution (8) can be expressed as

$$
\pi(\boldsymbol{\beta}, \sigma \mid \mathbf{y}, \mathbf{X}) \propto\left(\sigma^{-2}\right)^{\frac{n+1}{2}} \exp \left\{-\sigma^{-2} \frac{1}{2}(\mathbf{y}-\mathbf{X} \boldsymbol{\beta})^{\prime}(\mathbf{y}-\mathbf{X} \boldsymbol{\beta})\right\}, \boldsymbol{\beta} \in \mathrm{B}
$$

it is straightforward to deduce that $\sigma^{-2} \mid \boldsymbol{\beta}, \mathbf{y}, \mathbf{X}$ follows a gamma distribution, specifically:

$$
\sigma^{-2} \mid \boldsymbol{\beta}, \mathbf{y}, \mathbf{X} \sim G a\left(\frac{n+3}{2}, \frac{1}{2}(\mathbf{y}-\mathbf{X} \boldsymbol{\beta})^{\prime}(\mathbf{y}-\mathbf{X} \boldsymbol{\beta})\right)
$$

In order to obtain the distribution of $\boldsymbol{\beta} \mid \sigma, \mathbf{X}, \mathbf{y}$, let us consider $\hat{\boldsymbol{\beta}}=\left(\mathbf{X}^{\prime} \mathbf{X}\right)^{-1} \mathbf{X}^{\prime} \mathbf{y}$ and $\hat{\mathbf{u}}=\mathbf{y}-\mathbf{X} \hat{\boldsymbol{\beta}}$ (the estimator and the least-squares residuals of the unconstrained linear regression model). By taking into consideration that

$$
(\mathbf{y}-\mathbf{X} \boldsymbol{\beta})^{\prime}(\mathbf{y}-\mathbf{X} \boldsymbol{\beta})=\hat{\mathbf{u}}^{\prime} \hat{\mathbf{u}}+(\hat{\boldsymbol{\beta}}-\boldsymbol{\beta})^{\prime} \mathbf{X}^{\prime} \mathbf{X}(\hat{\boldsymbol{\beta}}-\boldsymbol{\beta}),
$$

the joint posterior distribution (8) can be written as:

$$
\pi(\boldsymbol{\beta}, \sigma \mid \mathbf{y}, \mathbf{X}) \propto\left(\sigma^{2}\right)^{-(n+1) / 2} \exp \left\{-\frac{\hat{\mathbf{u}} \cdot \hat{\mathbf{u}}}{2 \sigma^{2}}\right\} \exp \left\{-\frac{1}{2 \sigma^{2}}(\hat{\boldsymbol{\beta}}-\boldsymbol{\beta})^{\prime} \mathbf{X}^{\prime} \mathbf{X}(\hat{\boldsymbol{\beta}}-\boldsymbol{\beta})\right\}, \boldsymbol{\beta} \in \mathrm{B}
$$

from where it is obtained that 


$$
\boldsymbol{\beta} \mid \sigma, \mathbf{X}, \mathbf{y} \sim N_{k}\left(\hat{\boldsymbol{\beta}}, \sigma^{2}\left(\mathbf{X}^{\prime} \mathbf{X}\right)^{-1}\right), \boldsymbol{\beta} \in B
$$

that is, a k-dimensional normal distribution truncated to the subset $B \subseteq \mathbb{R}^{k}$.

In principle, a simple algorithm can be formulated through the following straightforward procedure:

Let $m$ be the sample step of the algorithm. Given the values $\boldsymbol{\beta}^{\mathbf{m}}$ and $\left(\sigma^{2}\right)^{m}$, obtained in the $m$-th step, the generated values in the next step are given by:

1. Generate a value $v^{m+1}$ of the distribution $\sigma^{-2} \mid \boldsymbol{\beta}^{\mathbf{m}}, \mathbf{y}, \mathbf{X}$. Take $\left(\sigma^{2}\right)^{m+1}=1 / v^{m+1}$.

2. a) Generate a value $\boldsymbol{\beta}^{*}$ from the distribution $\boldsymbol{\beta} \mid\left(\sigma^{2}\right)^{m+1}, \mathbf{y}, \mathbf{X}$, that is, generate a value

$$
\boldsymbol{\beta}^{*} \sim N_{k}\left(\hat{\boldsymbol{\beta}},\left(\sigma^{2}\right)^{m+1}\left(\mathbf{X}^{\prime} \mathbf{X}\right)^{-1}\right)
$$

b) If $\boldsymbol{\beta}^{*} \in B$, then take $\boldsymbol{\beta}^{\mathrm{m}+1}=\boldsymbol{\beta}^{*}$

$$
\text { If } \boldsymbol{\beta}^{*} \notin B \text {, then return to step 2.a) }
$$

However, we have found that, in practice, the probability of rejection in this procedure is frequently so high that the method becomes unviable.

In order to overcome this major difficulty, one solution is to apply the Gibbs algorithm by obtaining samples from the one-dimensional conditional distributions, that is, from $\sigma \mid \boldsymbol{\beta}, \mathbf{y}, \mathbf{X}$ and $\beta_{j} \mid \boldsymbol{\beta}_{(\mathrm{j})}, \sigma, \mathbf{y}, \mathbf{X}, \mathrm{j}=1, \ldots, \mathrm{k}$, where $\boldsymbol{\beta}_{(\mathrm{j})}=\left(\beta_{1}, \ldots, \beta_{j-1}, \beta_{j+1}, \ldots, \beta_{k}\right)^{\prime} \in \mathbb{R}^{k-1}$.

Therefore the Gibbs algorithm for this model is formulated as follows: Let $m$ be the sample step of the algorithm. Given the values $\boldsymbol{\beta}^{\mathrm{m}}$ and $\left(\sigma^{2}\right)^{m}$, obtained in the $m$-th step, the values generated in the next step are given by:

1. Generate a value $v^{m+1}$ from the distribution $\sigma^{-2} \mid \boldsymbol{\beta}^{\mathbf{m}}, \mathbf{y}, \mathbf{X}$. Take $\left(\sigma^{2}\right)^{m+1}=1 / v^{m+1}$.

2. $\quad$ FOR $\mathrm{j}=1 \mathrm{TO} \mathrm{k}$

Generate a value $\beta_{j}^{m+1}$ from the distribution

$$
\beta j \mid \beta_{1}^{m+1}, \ldots, \beta_{j-1}^{m+1}, \beta_{j+1}^{m}, \ldots, \beta_{k}^{m},\left(\sigma^{2}\right)^{m+1}, \mathbf{y}, \mathbf{X}
$$

\section{NEXT j}


3. Take $\boldsymbol{\beta}^{\mathbf{m + 1}}=\left(\beta_{1}^{m+1}, \ldots, \beta_{k}^{m+1}\right)^{\prime}$.

In order to obtain simulated samples for $\beta_{j} \mid \boldsymbol{\beta}_{(\mathrm{j})}, \sigma, \mathbf{y}, \mathbf{X}$, by rearranging the parameters, the following partition can be considered:

$$
\boldsymbol{\beta}=\left(\begin{array}{c}
\beta_{j} \\
\boldsymbol{\beta}_{(\mathrm{j})}
\end{array}\right) \text { and } \Sigma=\left(\begin{array}{cc}
\sigma_{j}^{2} & \boldsymbol{\sigma}_{\mathrm{j}(\mathrm{j})}^{\prime} \\
\boldsymbol{\sigma}_{\mathrm{j}(\mathrm{j})} & \boldsymbol{\Sigma}_{\mathbf{j}(\mathrm{j})}
\end{array}\right) \text {, }
$$

where $\boldsymbol{\Sigma}=\sigma^{2}\left(\mathbf{X}^{\prime} \mathbf{X}\right)^{-1}$. If there are no constraints in the model, it is known that

$$
\beta_{j} \mid \boldsymbol{\beta}_{(\mathrm{j})}, \sigma, \mathbf{y}, \mathbf{X} \sim N\left(\hat{\beta}_{j}+\Sigma_{\mathbf{j}(\mathrm{j})}^{-1}\left(\boldsymbol{\beta}_{(\mathrm{j})}-\hat{\boldsymbol{\beta}}_{(\mathrm{j})}\right), \sigma_{j}^{2}-\boldsymbol{\sigma}_{\mathbf{j}(\mathrm{j})}^{\prime} \boldsymbol{\Sigma}_{\mathbf{j}(\mathrm{j})}^{-1} \boldsymbol{\sigma}_{\mathbf{j}(\mathrm{j})}\right)
$$

For the sake of simplicity, let us assume initially that all the explanatory variables take nonnegative values. Hence, the constraints $y_{i} \leq \mathbf{x}_{\mathbf{i}}^{\prime} \boldsymbol{\beta}, i=1, \ldots, n$ are equivalent to

$$
\beta_{j} \geq \frac{y_{i}-\mathbf{x}_{\mathbf{i}(j)}^{\prime} \boldsymbol{\beta}_{(j)}}{x_{i j}}, i=1, \ldots, n \Leftrightarrow \beta_{j} \geq \max _{i}\left\{\frac{y_{i}-\mathbf{x}_{\mathbf{i j}(\mathbf{j})}^{\prime} \boldsymbol{\beta}_{(\mathrm{j})}}{x_{i j}}\right\} \Leftrightarrow \beta_{j} \geq b\left(\boldsymbol{\beta}_{(\mathrm{j})}, \mathbf{X}, \mathbf{y}\right)
$$

where $b\left(\boldsymbol{\beta}_{(\mathrm{j})}, \mathbf{X}, \mathbf{y}\right)=\max _{i}\left\{\left(y_{i}-\mathbf{x}_{\mathbf{i}(\mathrm{j})}^{\prime} \boldsymbol{\beta}_{(\mathrm{j})}\right) / x_{i j}\right\}$. (Note that if some $x_{i j}$ are zero, then the $i$-th observation does not take part in the set of constraints that the parameter $\beta_{j}$ must satisfy). In this way, it holds that

$$
\beta_{j} \mid \boldsymbol{\beta}_{(\mathrm{j})}, \sigma, \mathbf{y}, \mathbf{X} \sim N\left(\hat{\boldsymbol{\beta}}_{\mathbf{j}}+\boldsymbol{\Sigma}_{\mathbf{j}(\mathbf{j})}^{-1}\left(\boldsymbol{\beta}_{(\mathrm{j})}-\hat{\boldsymbol{\beta}}_{(\mathrm{j})}\right), \sigma_{j}^{2}-\boldsymbol{\sigma}_{\mathrm{j}(\mathrm{j})}^{\prime} \boldsymbol{\Sigma}_{\mathbf{j}(\mathbf{j})}^{-1} \boldsymbol{\sigma}_{\mathbf{j}(\mathrm{j})}\right), \beta_{j} \geq b\left(\boldsymbol{\beta}_{(\mathrm{j})}, \mathbf{X}, \mathbf{y}\right),
$$

that is, the one-dimensional conditional distributions are normal truncated to the interval $\left[b\left(\boldsymbol{\beta}_{(\mathrm{j})}, \mathbf{X}, \mathbf{y}\right),+\infty\right)$. In practice, it is frequent for this interval to have a very small probability, which renders the simulation by the acceptance-rejection method unfeasible. However, the simulation of values from this distribution can easily be performed by following the algorithm by Marsaglia, which is explained in Devroye (1986), p.380.

Specifically, this algorithm generates values from a distribution $Z \sim N(0,1)$ constrained to the range $z>a$, where $a>0$. The algorithm is as follows:

1. Generate $U, V$ independent values from a distribution $\mathrm{U}(0,1)$.

2. Calculate $Z=\sqrt{a^{2}-2 \log (U)}$.

3. If $V \cdot Z>a$ return to 1 . If $V \cdot Z \leq a$, take the value $Z$ as result. 
To apply this algorithm to our case, it is sufficient to take $Z$ in each step as the typified distribution of $\beta_{j} \mid \boldsymbol{\beta}_{(\mathrm{j})}, \sigma, \mathbf{y}, \mathbf{X}$, generate a value of $Z$, and then undo the typification.

In the framework of the production models, the variables are commonly measured in logarithmic terms and, as a consequence, negative values for the variables often appear.

If the hypothesis concerning the non-negativity of the explanatory variables is removed, then the conditional distribution is similar, but now the range of the parameter is given by $b_{1}\left(\boldsymbol{\beta}_{(\mathrm{j})}, \mathbf{X}, \mathbf{y}\right) \leq \beta_{j} \leq b_{2}\left(\boldsymbol{\beta}_{(\mathrm{j})}, \mathbf{X}, \mathbf{y}\right)$, where

$$
\begin{aligned}
& b_{1}\left(\boldsymbol{\beta}_{(\mathrm{j})}, \mathbf{X}, \mathbf{y}\right)=\max _{i \mid x_{i j}>0}\left\{\left(y_{i}-\mathbf{x}_{\mathbf{i}(\mathbf{j})}^{\prime} \boldsymbol{\beta}_{(\mathrm{j})}\right) / x_{i j}\right\} \\
& b_{2}\left(\boldsymbol{\beta}_{(\mathrm{j})}, \mathbf{X}, \mathbf{y}\right)=\min _{i \mid x_{i j}<0}\left\{\left(y_{i}-\mathbf{x}_{\mathrm{i}(\mathrm{j})}^{\prime} \boldsymbol{\beta}_{(\mathrm{j})}\right) / x_{i j}\right\} .
\end{aligned}
$$

If the set of indices $\left\{i \mid x_{i j}>0\right\}$ is empty, $b_{1}\left(\boldsymbol{\beta}_{(\mathrm{j})}, \mathbf{X}, \mathbf{y}\right)=-\infty$ is taken (similarly, if $\left\{i \mid x_{i j}<0\right\}=\varnothing$ then $\left.b_{2}\left(\boldsymbol{\beta}_{(\mathrm{j})}, \mathbf{X}, \mathbf{y}\right)=+\infty\right)$. In this way, when generating the values of the onedimensional conditional distributions $\beta_{j} \mid \boldsymbol{\beta}_{(\mathrm{j})}, \sigma, \mathbf{y}, \mathbf{X}$, a number of different situations appear depending on the values of $b_{1}\left(\boldsymbol{\beta}_{(\mathrm{j})}, \mathbf{X}, \mathbf{y}\right)$ and $b_{2}\left(\boldsymbol{\beta}_{(\mathrm{j})}, \mathbf{X}, \mathbf{y}\right)$. This entails additional complexity when programming the Gibbs algorithm, which can be solved, according to the cases, by utilizing the Marsaglia algorithm or applying the acceptance-rejection method.

As initial value $\boldsymbol{\beta}^{0}$, the corrected least-squares estimator is used (Green, 1980; Ortega et al., 2009), which consists of modifying one of the components of the unconstrained least-squares estimator so that the obtained point belongs to the frontier of the feasible region. Specifically, the estimation of the intercept of the model is modified, by replacing the first component of $\hat{\boldsymbol{\beta}}$ for $\hat{\beta}_{1}^{*}=\max _{i}\left\{y_{i}-\mathbf{x}_{\mathbf{i}(1)}^{\prime} \hat{\boldsymbol{\beta}}_{(1)}\right\}$.

\section{SOME SIMULATIONS}

First of all, a number of simple simulated examples are carried out, which helps towards ascertaining the formulation of the model and offers a first evaluation of the proposed estimation method. Despite the fact that the analysed model presents some complications, the Bayesian estimation with the help of the Gibbs algorithm offer a solution that is relatively straightforward to put into practice and, as will be seen, in general provide satisfactory results. 
Example 1. In order to better understand the nature of the problem and the complications of dealing with constraints involving both the parameters and the observations, we begin by considering a simple model, in which there is only one explanatory variable (plus an intercept), and a simulated sample of size $n=5$. Therefore, the regression model is $y_{i}=\beta_{1}+\beta_{2} x_{i}-\varepsilon_{i}, \quad i=1, \ldots, n$, where the hypotheses mentioned in Section 2 are assumed. The values $\beta_{1}=\beta_{2}=\sigma^{2}=1$ are chosen. For the variable $\mathbf{x}$, a sample of size 5 from a uniform model in $[0,1]$ is simulated. From these values, the corresponding sample for the variable $\mathbf{y}$ is simulated. The resulting sample is shown in the Appendix.

The constraints that the considered model imposes are $y_{i} \leq \beta_{1}+\beta_{2} x_{i}, i=1, \ldots, n$ or, equivalently, $\beta_{1} \geq y_{i}-\beta_{2} x_{i}, i=1, \ldots, n$, where in general there will be redundant constraints.

For the obtained sample, the ordinary least-squares estimator is $\hat{\beta}_{1}=0.3106$ and $\hat{\beta}_{2}=0.7938$, which does not belong to the feasible region and that greatly departs from the actual value for the case of the intercept. As explained in Section 3, it holds that $\boldsymbol{\beta} \mid \sigma, \mathbf{X}, \mathbf{y} \sim N_{2}\left(\hat{\boldsymbol{\beta}}, \sigma^{2}\left(\mathbf{X}^{\prime} \mathbf{X}\right)^{-1}\right), \boldsymbol{\beta} \in B$, where B is the set of points belonging to the feasible region. Therefore, the marginal posterior distribution of $\boldsymbol{\beta}$ concentrates a greater probability in the zone of the frontier of the feasible region nearer to the point given by $\hat{\boldsymbol{\beta}}$.

In Figure 1, a scatter plot corresponding to a simulated sample of size 2000 of the distribution of $\boldsymbol{\beta} \mid \sigma, \mathbf{X}, \mathbf{y}$ is offered, together with the straight lines that define the feasible region. Indeed, the points accumulate in the zone of greater probability. As mentioned earlier, some constraints can be redundant with some others (as is the case of the constraint in the bottom left-hand corner).

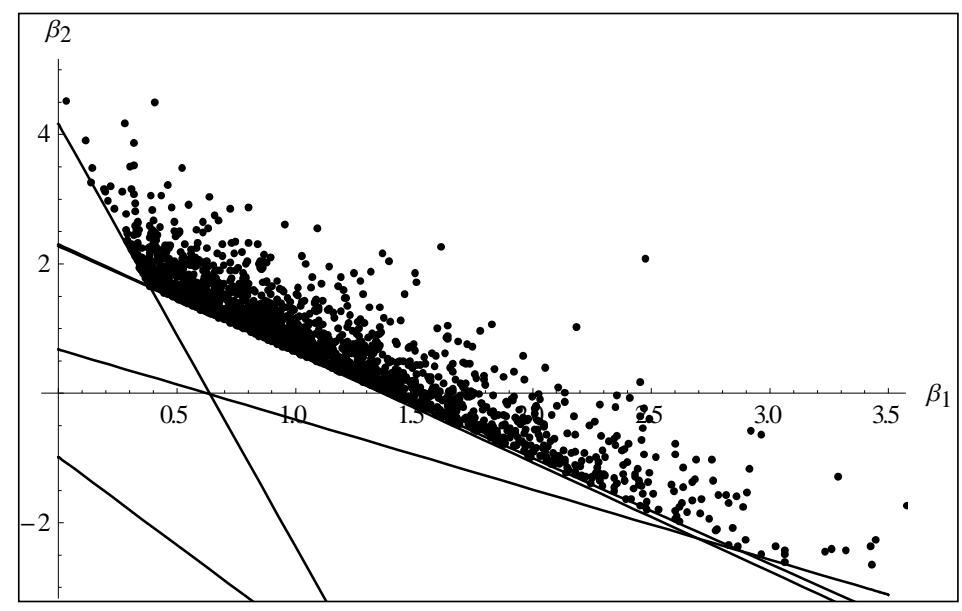

Fig. 1: Simulated sample of the posterior distribution (Example 1). 
In order to estimate the values of the parameters, the criterion of the mathematical expectation of the posterior marginal distributions is used. Therefore, the average of each of the components of the simulated sample is taken (it is well-known that they converge towards the mathematical expectations as the size of the simulated sample tends towards infinity). In the same way, estimations of the standard errors are obtained from the standard deviations of the simulated sample and the symmetrical probabilistic intervals are obtained from the quantiles of order 0.025 and 0.975 of the simulated sample (Chen et al., 2000).

For the considered samples, the Bayesian estimation of the parameters is $\hat{\beta}_{1}^{B}=1.0924$ and $\hat{\beta}_{2}^{B}=0.8605$; the standard errors are $S E\left(\hat{\beta}_{1}^{B}\right)=0.6036$ and $S E\left(\hat{\beta}_{2}^{B}\right)=1.0433$, while the symmetric probabilistic (or credible) intervals are $\operatorname{SPI}\left(\beta_{1}\right)=(0.3514,2.5636)$ and $\operatorname{SPI}\left(\beta_{2}\right)=(-1.5419,2.5196)$. It should be emphasised that, in this case, the estimations are not very accurate because a very small sample size is considered.

As a consequence of the constraints involving the parameters and the observations, in this type of model the probability density function of the joint posterior distribution is not continuous at the frontier, and therefore certain graphical representations (such as the threedimensional histogram and the contour plot) are not suitable, since they underestimate the true value of the density at the frontier. That is the reason why the scatter plot in Figure 1 has been shown. In order to have a better understanding of the posterior distribution, in Figure 2, the smoothed histograms of the marginal posterior distributions are presented. It is observed that the two distributions are skew and bell-shaped and have the highest density around the true values of the parameters.

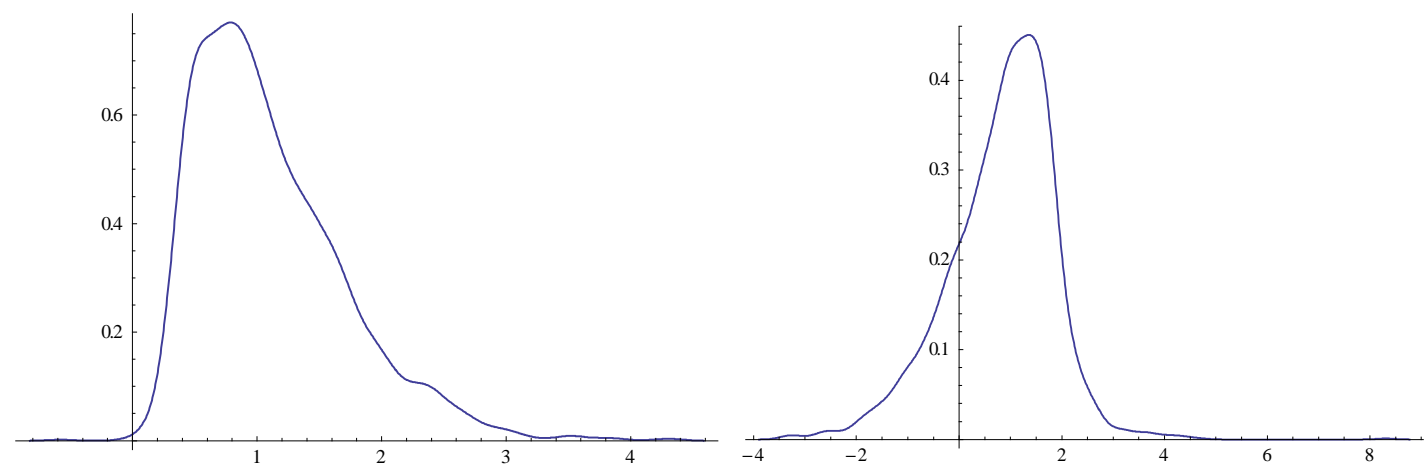

Fig. 2: Smooth Histogram of the posterior distribution of $\beta_{1}$ (left) and $\beta_{2}$ (right) of Example 1.

Example 2. A model with two explanatory variables and an intercept is now considered, for which a simulated sample of size $n=50$ is obtained. Therefore, the regression model is $y_{i}=\beta_{1}+\beta_{2} x_{i 2}+\beta_{3} x_{i 3}-\varepsilon_{i}, \quad i=1, \ldots, n$, where the hypotheses mentioned in Section 2 are 
assumed. Independent samples of size 50 from the uniform model in $[0,1]$ are simulated for the variables $\mathbf{x}_{2}$ and $\mathbf{x}_{3}$. Taking $\beta_{1}=\beta_{2}=\beta_{3}=\sigma^{2}=1$, the corresponding sample for the variable $\mathbf{y}$ is simulated. The resulting sample is shown in the Appendix.

For the considered set of data, the ordinary least-squares estimator is the vector $\hat{\boldsymbol{\beta}}=(0.5632,0.7837,0.2729)^{\prime}$, which does not belong to the feasible region and is far from the true value of the parameter, especially for the case of the intercept and the second slope. Through the Gibbs algorithm, a simulated sample of size 2000 for the distribution of $\boldsymbol{\beta} \mid \sigma, \mathbf{X , \mathbf { y }}$ is obtained, whose scatter plot is shown in Figure 3, where the zone of the feasible region with the highest probability can be observed.

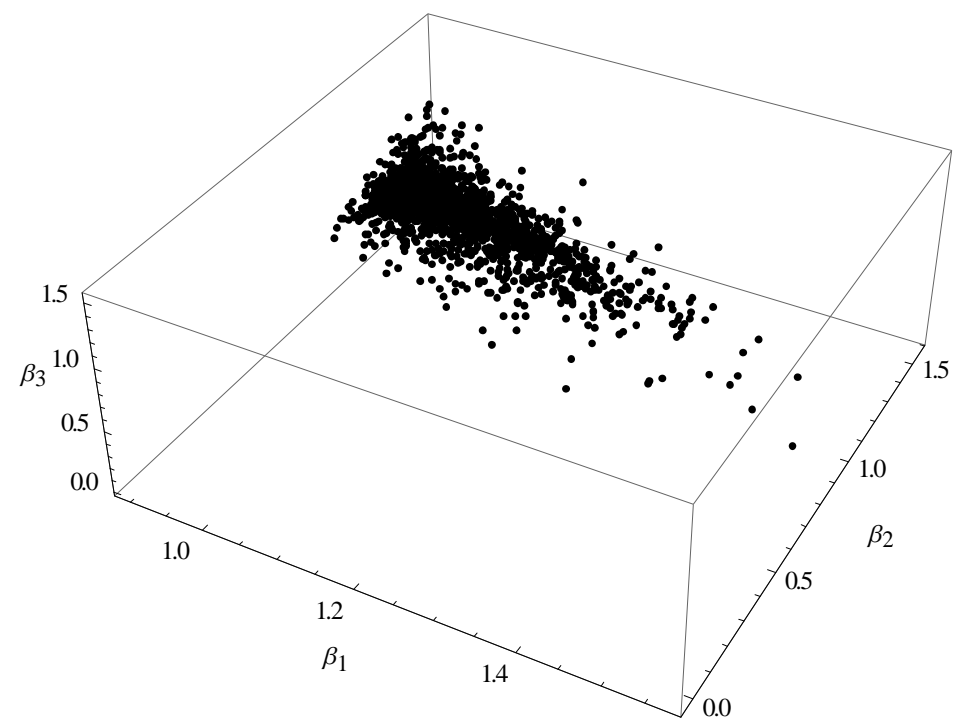

Fig. 3: Simulated sample of the posterior distribution (Example 2).

For the sample generated, the results obtained by applying the Bayesian DFM are shown in Table 1. As can be noted, for this sample of moderate size, the accuracy of the estimator is acceptable. In Figure 4, the smoothed histograms of the marginal posterior distributions are presented, where it is observed that the highest densities are located around the true values of the parameters. The results obtained by using maximum likelihood in the SFM are also shown in Table 1. Since the considered sample has been generated from a deterministic frontier model, an estimation of the parameter gamma equal (or near) to one is expected. However, the estimation obtained is, surprisingly, practically zero $(\hat{\gamma}=0.000047)$. Therefore, instead of identifying the deterministic frontier model, the SFM estimates, on the contrary, a stochastic frontier model without inefficiency, that is to say, all the composite error is assigned to noise. As a result of being unable to identify the true structure of the composite error of the model, the estimations of the parameters are very far from their true values. In this situation, all the 
efficiencies are estimated to be practically 1 (the maximum attainable level of efficiency) in the SFM (all are above 0.995). For the case of the Bayesian DFM, the estimated average efficiency is 0.4587 , which is near to the average of the true efficiencies, 0.4751 . The average absolute of the differences between the true efficiencies and the efficiencies estimated through the Bayesian DFM is 0.018 (where the largest difference is 0.055), and their Pearson and Spearman rank coefficients of correlations are 0.9986 and 0.9973 , respectively, again indicating reliable estimations. To conclude, in this example the SFM approach is unable to identify the true structure of the composite error and therefore provides unreliable estimations of the parameters and of the efficiencies. The Bayesian DFM approach, however, provides accurate estimations.

Table 1: Results of the Bayesian DFM and SFM estimations for the simulated sample of Example 2.

\begin{tabular}{|c|c|c|c|c|c|}
\hline \multicolumn{5}{|c|}{ Bayesian DFM } & \multicolumn{2}{c|}{ SFM } \\
\hline Parameter & Estimation & Std. Error & SPI & Estimation & Std. Error \\
\hline$\beta_{0}$ & 1.05907 & 0.090510 & $(0.948005,1.29077)$ & 0.566297 & 1.159324 \\
\hline$\beta_{1}$ & 1.04719 & 0.176417 & $(0.627511,1.31936)$ & 0.783662 & 0.267659 \\
\hline$\beta_{2}$ & 0.90686 & 0.194569 & $(0.529324,1.31864)$ & 0.272940 & 0.283047 \\
\hline$\sigma^{2}$ & 1.23846 & 0.272368 & $(0.822994,1.86607)$ & 0.311541 & 0.063564 \\
\hline
\end{tabular}
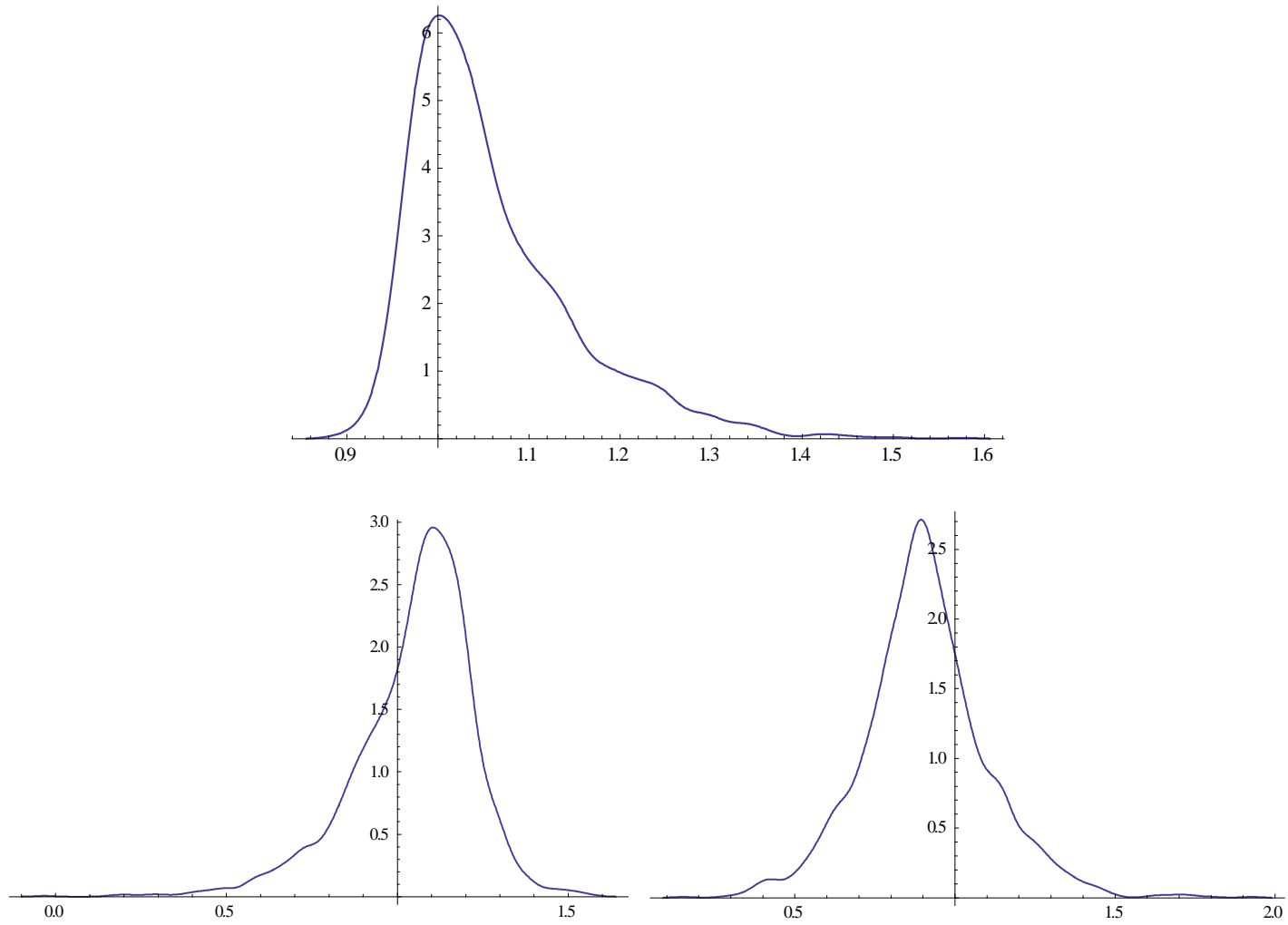

Fig. 4: Smooth Histogram of the posterior distribution of $\beta_{1}$ (upper), $\beta_{2}$ (bottom left) and $\beta_{3}$ (bottom right) of Example 2. 
Similar examples, with a greater number of explanatory variables and with different starting values for the parameters, have been analysed. It has been observed that the accuracy of the estimators remains unaltered. Generally speaking, in the setting of the regression models with frontier (both deterministic and stochastic), the greatest difficulty is the estimation of the intercept; as a matter of fact, the ordinary least square estimators of the slopes are consistent (Green, 1980). The number of explanatory variables in the model and the values of the slopes are not determining factors in the statistical properties of the estimators of the intercept and the efficiencies. In this sense, in Coelli (1995), a simulation analysis for stochastic frontier models is carried out in which there is only one intercept and no explanatory variables, and it is argued that the main results remain unchanged when including explanatory variables. In Olsen et al. (1980), it is also observed that the inclusion of four explanatory variables in the stochastic frontier model provides very similar results to those of the model containing only the intercept.

The following is a Monte Carlo analysis, in which, for each of the chosen values of $n, 1000$ samples of size $n$ are generated for the considered model. To these samples, the Gibbs algorithm with samples of size 2000 is applied, with the objective of studying the bias and the mean squared error (MSE) of the estimators, the length (Len) of the symmetrical intervals of probability 0.95 (SPI), and the proportion of success of such intervals (Succ), that is, the frequentist coverage probability of the symmetrical Bayesian intervals. For the case of the estimated efficiencies, their averages for each of the observations are obtained.

A model with one explanatory variable and an intercept is selected, together with the values $\beta_{1}=\beta_{2}=\sigma^{2}=1$. With the intention of analysing the possible influence of the dispersion in the set of data of the explanatory variables on the results, the procedure is carried out twice: the first time, the values for the variable $\mathbf{x}$ are simulated from the model $U(0,1)$; while the second time, the model $U(0,5)$ is used instead. The results obtained are shown in Tables 2 and 3, respectively.

Table 2: Properties of the estimators and probabilistic intervals $\left(\beta_{1}=\beta_{2}=\sigma^{2}=1, x_{i} \sim U(0,1)\right)$.

\begin{tabular}{|c|c|c|c|c|c|c|c|c|c|c|}
\hline & \multicolumn{5}{|c|}{$\beta_{1}$} & \multicolumn{5}{|c|}{$\beta_{2}$} \\
\hline $\mathbf{n}$ & Bias & MSE & SPI & Len & Succ & Bias & MSE & SPI & Len & Succ \\
\hline 10 & -0.0297 & 0.0746 & $(0.583,1.557)$ & 0.974 & 0.912 & 0.0051 & 0.2736 & $(0.067,1.948)$ & 1.881 & 0.904 \\
\hline 20 & -0.0074 & 0.0213 & $(0.779,1.337)$ & 0.558 & 0.944 & 0.0047 & 0.0909 & $(0.429,1.621)$ & 1.193 & 0.948 \\
\hline 50 & -0.0020 & 0.0060 & $(0.895,1.178)$ & 0.283 & 0.948 & 0.0000 & 0.0173 & $(0.748,1.258)$ & 0.509 & 0.944 \\
\hline \multirow[t]{2}{*}{100} & 0.0001 & 0.0019 & $(0.949,1.109)$ & 0.159 & 0.948 & -0.0003 & 0.0055 & $(0.865,1.134)$ & 0.269 & 0.946 \\
\hline & \multicolumn{5}{|c|}{$\sigma^{2}$} & \multicolumn{5}{|c|}{ Efficiencies (averages) } \\
\hline $\mathbf{n}$ & Bias & MSE & SPI & Len & Succ & Bias & MSE & SPI & Length & Succ \\
\hline 10 & -0.1109 & 0.2383 & $(0.346,2.143)$ & 1.797 & 0.856 & 0.0178 & 0.0113 & $(0.352,0.658)$ & 0.306 & 0.908 \\
\hline 20 & -0.0529 & 0.1219 & $(0.489,1.768)$ & 1.279 & 0.904 & 0.0041 & 0.0035 & $(0.410,0.599)$ & 0.190 & 0.938 \\
\hline
\end{tabular}




\begin{tabular}{|c||c|c|c|c|c||c|c|c|c|c|}
\hline 50 & -0.0297 & 0.0419 & $(0.660,1.449)$ & 0.790 & 0.928 & 0.0013 & 0.0008 & $(0.465,0.552)$ & 0.088 & 0.951 \\
\hline 100 & -0.0099 & 0.0210 & $(0.737,1.297)$ & 0.560 & 0.938 & 0.0008 & 0.0002 & $(0.495,0.541)$ & 0.047 & 0.947 \\
\hline
\end{tabular}

In relation to the bias, the values are, in general, close to 0 (with the exception of the parameter $\sigma$ for $n=10$ ), and are smaller, in absolute values, as the sample size increases. Therefore, it can be concluded that the estimators have a good behaviour with respect to the estimation bias. In addition, it should be noted that the parameters $\beta_{1}$ and $\sigma^{2}$ present persistence of negative bias while $\beta_{2}$ and the efficiencies present positive bias in almost all the cases.

With regard to the MSE and the length of the intervals, reasonable values are obtained. In general, as expected, these two quantities decrease as the sample size increases.

On the other hand, it can be observed that the frequentist coverage probability of the Bayesian intervals is close to their nominal probability (0.95) for sample sizes of 20 or over except for the case of the parameter $\sigma^{2}$, in which the values are maintained a little below the nominal value. For small sample sizes ( $n=10$ ), the coverage probability is less than 0.95 in all cases.

The simulation analysis carried out also shows that the estimators of the parameters $\beta_{1}$ and $\beta_{2}$, and of the efficiencies, have a better behaviour than the estimator of the parameter $\sigma^{2}$.

Table 3: Properties of the estimators and probabilistic intervals $\left(\beta_{1}=\beta_{2}=\sigma^{2}=1, x_{i} \sim U(0,5)\right)$

\begin{tabular}{|c|c|c|c|c|c|c|c|c|c|c|}
\hline & \multicolumn{5}{|c|}{$\beta_{1}$} & \multicolumn{5}{|c|}{$\beta_{2}$} \\
\hline $\mathbf{n}$ & Bias & MSE & SPI & Len & Succ & Bias & MSE & SPI & Len & Succ \\
\hline 10 & -0.0289 & 0.0455 & $(0.553,1.292)$ & 0.739 & 0.930 & 0.0005 & 0.0055 & $(0.807,1.081)$ & 0.275 & 0.922 \\
\hline 20 & -0.0036 & 0.0283 & $(0.758,1.350)$ & 0.591 & 0.934 & -0.0015 & 0.0032 & $(0.888,1.097)$ & 0.209 & 0.936 \\
\hline 50 & -0.0034 & 0.0054 & $(0.896,1.162)$ & 0.266 & 0.952 & -0.0001 & 0.0008 & $(0.952,1.050)$ & 0.098 & 0.946 \\
\hline \multirow[t]{2}{*}{100} & 0.0005 & 0.0012 & $(0.951,1.081)$ & 0.1306 & 0.950 & -0.0004 & 0.0002 & $(0.973,1.026)$ & 0.053 & 0.958 \\
\hline & \multicolumn{5}{|c|}{$\sigma^{2}$} & \multicolumn{5}{|c|}{ Efficiencies (averages) } \\
\hline $\mathrm{n}$ & Bias & MSE & SPI & Len & Succ & Bias & MSE & SPI & Len & Succ \\
\hline 10 & -0.1313 & 0.2406 & $(0.343,2.114)$ & 1.771 & 0.842 & 0.0179 & 0.0106 & $(0.352,0.651)$ & 0.299 & 0.926 \\
\hline 20 & -0.0832 & 0.1099 & $(0.488,1.725)$ & 1.237 & 0.890 & 0.0052 & 0.0036 & $(0.410,0.597)$ & 0.187 & 0.933 \\
\hline 50 & -0.0226 & 0.0431 & $(0.659,1.455)$ & 0.7957 & 0.930 & 0.0022 & 0.0008 & $(0.466,0.554)$ & 0.088 & 0.949 \\
\hline 100 & -0.0073 & 0.0197 & $(0.766,1.327)$ & 0.561 & 0.948 & 0.0002 & 0.0002 & $(0.489,0.536)$ & 0.047 & 0.947 \\
\hline
\end{tabular}

The effect of the variability of $\mathbf{x}$ is clearly manifest in the MSE and the length of the interval of the parameter $\beta_{2}$; as expected, these two quantities significantly decrease as the dispersion of the explanatory variable increases. It can also be observed that any increase in the variability of $\mathbf{x}$ has no appreciable effect on the other parameters nor on the efficiencies. 
Finally, in order to analyse the influence of the variance of the perturbation $\varepsilon$ on the estimators, the simulation study has again been made selecting $\beta_{1}=\beta_{2}=1$ and $\sigma^{2}=9$, and taking values for $\mathbf{x}$ from distributions $U(0,1)$ and $U(0,5)$. The results for $\mathbf{x} \sim U(0,1)$ are shown in Table 4; similar results are obtained for $\mathbf{x} \sim U(0,5)$ and are therefore omitted.

Table 4: Properties of the estimators and probabilistic intervals $\left(\beta_{1}=\beta_{2}=\sigma^{2}=9, x_{i} \sim U(0,1)\right)$.

\begin{tabular}{|c||c|c|c|c|c||c|c|c|c|c|}
\hline \multicolumn{1}{|c||}{} & \multicolumn{9}{c||}{$\beta_{1}$} & \multicolumn{3}{|c|}{$\beta_{2}$} \\
\hline $\mathbf{n}$ & Bias & MSE & SPI & Len & Succ & Bias & MSE & SPI & Len & Succ \\
\hline 10 & -0.1318 & 0.8989 & $(-0.280,2.840)$ & 3.120 & 0.906 & 0.034 & 2.764 & $(-1.820,3.876)$ & 5.696 & 0.902 \\
\hline 20 & -0.0422 & 0.2653 & $(0.277,2.091)$ & 1.814 & 0.924 & 0.0032 & 0.8939 & $(-0.693,2.721)$ & 3.414 & 0.922 \\
\hline 50 & -0.0090 & 0.0528 & $(0.695,1.511)$ & 0.816 & 0.930 & 0.0028 & 0.1559 & $(0.239,1.755)$ & 1.516 & 0.942 \\
\hline 100 & -0.0030 & 0.0159 & $(0.844,1.267)$ & 0.423 & 0.950 & 0.0049 & 0.0540 & $(0.607,1.396)$ & 0.789 & 0.943 \\
\hline \hline & \multicolumn{9}{|c|}{$\sigma^{2}$} & \multicolumn{7}{|c|}{ Efficiencies (averages) } \\
\hline $\mathbf{n}$ & Bias & MSE & SPI & Len & Succ & Bias & MSE & \multicolumn{1}{|c|}{ SPI } & Length & Succ \\
\hline 10 & -1.3386 & 17.5966 & $(3.027,18.554)$ & 15.527 & 0.824 & 0.0298 & 0.0226 & $(0.0824,0.4387)$ & 0.3563 & 0.917 \\
\hline 20 & -0.7046 & 8.077 & $(4.365,15.547)$ & 11.182 & 0.924 & 0.0108 & 0.0096 & $(0.1185,0.3487)$ & 0.230 & 0.926 \\
\hline 50 & -0.1916 & 3.6670 & $(5.913,13.079)$ & 7.166 & 0.928 & 0.0019 & 0.0023 & $(0.173,0.288)$ & 0.115 & 0.947 \\
\hline 100 & -0.0889 & 1.7619 & $(6.732,11.772)$ & 5.039 & 0.914 & 0.0001 & 0.0007 & $(0.202,0.265)$ & 0.063 & 0.943 \\
\hline
\end{tabular}

As expected, the increase of the variance of the perturbations has the effect of decreasing the accuracy of the estimators of the parameters $\beta_{1}, \beta_{2}$ and $\sigma^{2}$, and of making the bias, the MSE and the length of the probabilistic intervals larger. No influence on the coverage probabilities is observable.

For the case of the bias, since its absolute values are small, no clear factor of increase is visible. When analysing the parameters $\beta_{1}$ and $\beta_{2}$, it can be appreciated that the length of the probabilistic intervals increases by an approximate factor of 3, while the MSE increases by an approximate factor of 9 . These results are consistent with those for the ordinary least square estimator in the usual lineal regression model. The length of the probabilistic interval for $\sigma^{2}$ is approximately multiplied by 9 and its MSE by 81 . In relation to the efficiencies, no significant variations are produced in the bias, nor in the MSE, nor in the length of the probabilistic intervals for the averages. Clearly, there is a decrease in the endpoints of such intervals, because when increasing $\sigma$, the average inefficiency increases and therefore the efficiency decreases.

\section{TWO WELL-KNOWN EXAMPLES REVISTED}

In this section, the estimation method analysed is applied to two well-known databases in the literature on the frontier production models. The first set of data, considered in Whiteman and Pearson (1993) and in Coelli et al. (1998), refers to telecommunication companies belonging to 21 countries in 1990. A single output $y$ is considered, (which is an index based on incomes) 
and two inputs: the capital factor $x_{1}$ (measured through the kilometres of the lines) and the labour factor $x_{2}$ (number of employees). The set of data is shown in the Appendix.

In this framework, we estimate, following the proposed methodology, a Cobb-Douglas production model, that is, $\mathbf{y}=\beta_{0}+\beta_{1} \mathbf{x}_{1}+\beta_{2} \mathbf{x}_{2}-\boldsymbol{\varepsilon}$, where the variables $\mathbf{y}, \mathbf{x}_{1}, \mathbf{x}_{2}$ are measured in logarithms and the perturbation $\boldsymbol{\varepsilon}$ follows a half-normal distribution $\left(0, \sigma^{2}\right), \sigma>0$.

The estimations of the parameters, together with their standard errors and symmetrical Bayesian intervals with probability 0.95 , are shown in Table 5 . As a starting point for the Gibbs algorithm, the unrestricted least-squares estimator is utilized, whose first component is modified so that it belongs to the frontier of the feasible region. A sample of size 10,000 from the joint posterior distribution is generated, from which the estimations offered in Table 5 are obtained. The results obtained in Coelli et al. (1998) are also shown in Table 5, where the parameters are estimated using maximum likelihood in a stochastic frontier production model. On page 198, they point out that "These results indicate that the vast majority of residual variation is due to the inefficiency effect, $u_{i}$, and that the random error, $v_{i}$, is approximately zero", that is, that the frontier is actually deterministic. Therefore, the warning and error messages mentioned in the introduction are shown when using specific software to obtain the estimations of the model.

Table 5: Results of the estimation of the telecommunications model.

\begin{tabular}{|c|c|c|c|c|c|}
\hline \multicolumn{5}{|c|}{ Bayesian DFM } & \multicolumn{2}{c|}{ SFM } \\
\hline Parameter & Estimation & Std. Error & Interval & Estimation & Std. Error \\
\hline$\beta_{0}$ & 0.221224 & 0.088662 & $(0.098279,0.429807)$ & 0.170302 & 0.084008 \\
\hline$\beta_{1}$ & 0.923822 & 0.193276 & $(0.466299,1.254850)$ & 0.968427 & 0.216607 \\
\hline$\beta_{2}$ & -0.075194 & 0.176745 & $(-0.402556,0.322700)$ & -0.100169 & 0.197732 \\
\hline$\sigma^{2}$ & 0.376945 & 0.127413 & $(0.198190,0.670106)$ & 0.286561 & 0.077963 \\
\hline
\end{tabular}

As can be observed, all the results achieved using SFM are similar to those obtained here by means of the Bayesian DFM. The largest discrepancy is observed in the parameter $\sigma^{2}$, both in the estimation and in the standard error, where the absolute difference is approximately 0.09 for the estimation and 0.05 for the standard error. For the parametric vector $\boldsymbol{\beta}$, the largest difference for the estimations is 0.05 (for the intercept) and for the standard error, this is 0.02 (for $\beta_{1}$ ).

In Table 6, the estimated efficiency is shown for each country, together with its standard errors and probabilistic intervals obtained using the Bayesian DFM. The estimated efficiencies calculated in Coelli et al. (1998) using the SFM are also shown. Only the estimated efficiencies are attained because, in principle, the maximum likelihood estimation of the SFM allows neither the standard errors nor the confidence intervals of the estimated efficiencies to be obtained. 
Table 6: Efficiency of each of the countries.

\begin{tabular}{|c|c|c|c|c|}
\hline & \multicolumn{3}{|c|}{ Bayesian DFM } & SFM \\
\hline Country & Efficiency & Std. Deviation & Interval & Efficiency \\
\hline Australia & 0.743777 & 0.072733 & $(0.575758,0.859353)$ & 0.784300 \\
\hline Austria & 0.482098 & 0.026884 & $(0.410351,0.511599)$ & 0.510347 \\
\hline Belgium & 0.610151 & 0.030610 & $(0.530624,0.651875)$ & 0.645905 \\
\hline Canada & 0.685817 & 0.041381 & $(0.585526,0.746132)$ & 0.707590 \\
\hline Denmark & 0.860641 & 0.047756 & $(0.742071,0.924397)$ & 0.915252 \\
\hline Finland & 0.699179 & 0.047142 & $(0.594844,0.773231)$ & 0.747832 \\
\hline France & 0.660550 & 0.061755 & $(0.536390,0.790499)$ & 0.668246 \\
\hline Germany & 0.533938 & 0.043088 & $(0.426544,0.606291)$ & 0.543229 \\
\hline Iceland & 0.702297 & 0.170060 & $(0.358880,0.985397)$ & 0.788019 \\
\hline Ireland & 0.654088 & 0.106575 & $(0.429755,0.825631)$ & 0.715014 \\
\hline Italy & 0.573716 & 0.051907 & $(0.474924,0.684528)$ & 0.582440 \\
\hline Japan & 0.507945 & 0.063716 & $(0.395931,0.651712)$ & 0.504949 \\
\hline The Netherlands & 0.796301 & 0.060814 & $(0.647231,0.911924)$ & 0.825264 \\
\hline New Zealand & 0.664976 & 0.089423 & $(0.472417,0.807720)$ & 0.722120 \\
\hline Norway & 0.784314 & 0.054093 & $(0.663300,0.867537)$ & 0.841189 \\
\hline Portugal & 0.516446 & 0.051210 & $(0.404777,0.597854)$ & 0.555615 \\
\hline Spain & 0.374454 & 0.022856 & $(0.321480,0.413652)$ & 0.386433 \\
\hline Sweden & 0.876195 & 0.044082 & $(0.769552,0.940367)$ & 0.922833 \\
\hline Switzerland & 0.947784 & 0.050564 & $(0.809997,0.998708)$ & 0.999471 \\
\hline Turkey & 0.157315 & 0.009541 & $(0.132828,0.172900)$ & 0.163748 \\
\hline UK & 0.914882 & 0.074745 & $(0.714045,0.997120)$ & 0.939083 \\
\hline
\end{tabular}

With regard to the efficiencies, it is also observed that the results are very similar. The estimated average efficiency using the Bayesian DFM is 0.654613 while it is estimated to be 0.688994 when using the SFM. The estimated efficiencies using the Bayesian DFM are slightly lower than those obtained by utilising the SFM, however the average absolute difference is approximately 0.03 , with the largest difference approximately 0.08 (for Iceland). Overall, the Pearson correlation coefficient for the estimations obtained for the two methodologies is 0.995364; and if solely the rank of the efficiencies is considered, then a Spearman rank correlation equal to 0.988312 is obtained. In both cases, the correlations are almost perfect.

The second set of data refers to 247 dairy farms located in Northern Spain, in the year 1997. The output variable $y$ is given by the production of milk (in litres). Four inputs are considered: the total farm area $x_{1}$ (in hectares), the total labour, including hired and family labour $x_{2}$ (in worker equivalents), the total amount of feedstuffs fed to the dairy cows $x_{3}$ (in kilograms) and the average number of milking cows $x_{3}$. Additional information about the variables can be found in Cuesta (2000) and Alvarez and Arias (2004). 
In this setting, we estimate, according to the proposed methodology, a Cobb-Douglas production model, that is, $\mathbf{y}=\beta_{0}+\beta_{1} \mathbf{x}_{1}+\beta_{2} \mathbf{x}_{2}+\beta_{3} \mathbf{x}_{3}+\beta_{4} \mathbf{x}_{4}-\boldsymbol{\varepsilon}$, where the variables $\mathbf{y}, \mathbf{x}_{1}, \mathbf{x}_{2}$, $\mathbf{x}_{3}, \mathbf{x}_{4}$ are measured in logarithms, and the perturbation $\boldsymbol{\varepsilon}$ follows a half-normal distribution $\left(0, \sigma^{2}\right), \sigma>0$.

The estimations of the parameters, together with their standard errors and symmetrical Bayesian intervals with probability 0.95 are shown in Table 7 . The starting point for the Gibbs algorithm and the sample of size has been selected as in the previous example. The results obtained by using maximum likelihood in the SFM are also shown in Table 7.

Table 7: Results of the estimation of the Spanish dairy farm model.

\begin{tabular}{|c|c|c|c|c|c|}
\hline \multicolumn{5}{|c|}{ Bayesian DFM } & \multicolumn{2}{c|}{ SFM } \\
\hline Parameter & Estimation & Std. Error & Interval & Estimation & Std. Error \\
\hline$\beta_{0}$ & 5.59068 & 0.0992867 & $(5.31677,5.71072)$ & 5.0939209 & 0.1721667 \\
\hline$\beta_{1}$ & 0.0632154 & 0.0294959 & $(0.0226294,0.12624)$ & 0.0514371 & 0.0262519 \\
\hline$\beta_{2}$ & 0.0483018 & 0.0323639 & $(-0.0312353,0.0962546)$ & 0.0600841 & 0.0285625 \\
\hline$\beta_{3}$ & 0.413672 & 0.0157697 & $(0.393733,0.447925)$ & 0.4468382 & 0.0238105 \\
\hline$\beta_{4}$ & 0.55758 & 0.0157742 & $(0.522018,0.581791)$ & 0.5733713 & 0.0438262 \\
\hline$\sigma^{2}$ & 0.0884606 & 0.0083477 & $(0.0733932,0.1055258)$ & 0.0404724 & 0.0059082 \\
\hline
\end{tabular}

As can be appreciated, the results are similar in the two approaches. However, the estimation of the parameter $\gamma$ in the stochastic frontier setting is 0.8751 , indicating that there is a stochastic frontier. This means that the Bayesian approach for the studied case of deterministic frontier is robust and performs reasonably well in the setting of a stochastic frontier with a high value of the parameter $\gamma$ (the variance of the inefficiency term is much larger than the variance of the noise). For the estimations, the largest absolute difference between the two approaches is 0.4968 and is observed in the intercept; all the other absolute differences remain below 0.05 . In relation to the standard errors, the largest absolute difference between the two approaches is 0.0729 and it is again observed in the intercept; all the other absolute differences remain below 0.03. With regard to the estimated efficiencies, the results are also similar. The estimated mean efficiency through DFM is 0.7756 and through SFM this is slightly higher, at 0.8657 , due to the fact that, in the DFM framework, all the random error in the model is assigned to inefficiency while in the SFM case a little part of the error is random noise, and hence the global inefficiency is higher in the DFM framework, in other words, the global efficiency is lower. The average absolute difference between the estimated efficiencies in the two approaches is 0.0948, while the Pearson correlation is 0.8653 and the Spearman rank correlation is 0.8817 , thereby indicating a high degree of concordance between the two approaches. In conclusion, in general, similar results are obtained, indicating the robustness of the Bayesian DFM approach. However, 
as the frontier in this case is estimated to be stochastic, the differences between the two approaches are larger than in the previous example, in which the frontier is estimated to be deterministic.

\section{CONCLUSIONS}

The literature on frontier models has, in general, paid little attention to the DFM since the appearance of the SFM. However, the formulation using a deterministic frontier is simpler and enables exact results to be attained for the inference based on ML estimation in a number of cases (Amsler et al., 2013). The deterministic frontier model can be particularly useful in those situations where the effects of the inefficiency and the random noise in the SFM are complicated (and even "virtually impossible") to disentangle, especially when the estimation of the SFM suggests that the entire variance of the composite error is due to inefficiency.

One of the main drawbacks of the DFM is that it poses a non-regular estimation problem (using the most common probability distributions for the inefficiency term), which renders the inference through maximum likelihood difficult. It has been shown that the formulation of the Gibbs algorithm for the estimation of the model using the Bayesian methodology is relatively straightforward and that it holds a number of advantages in comparison with other estimation methods, such as dealing with constraints involving both the parameters and the observations (which make the model non-regular), and the possibility of making inferences in a direct way concerning the individual efficiencies. Furthermore, as the kernel of the exact posterior distribution is used, the approximations to the exact results depend on the size of the simulated sample in the Gibbs algorithm, which can be as large as desired (in the maximum likelihood framework, the approximations depend on the sample size, which is fixed).

Both the simulation analysis and the examples considered show a good behaviour of the proposed methodology. For moderate sample sizes, the biases and the mean squared errors are considerably small and the frequentist coverage probability of the Bayesian intervals is practically identical to their nominal probability.

\section{REFERENCES}

Aigner, DJ, Chu SF (1968) On estimating the industry production function. Am Econ Rev 58: 826-839.

Aigner DJ, Lovell CA, Schmidt P (1977) Formulation and estimation of stochastic frontier production function models. J Econom 6: 21-37.

Alvarez A, Arias C (2004) Technical efficiency and farm size: a conditional analysis. Agr Econ 30: 241250. 
Amsler C, Leonard M, Schmidt P (2013) Estimation and inference in parametric deterministic frontier models. J Prod Anal 40: 293-305

Battese GE, Corra GS (1977) Estimation of a production frontier model: With application to the Pastoral Zone of Eastern Australia. Aust J Agr Econ 21: 169-179.

Box EP, Tiao GC (1973) Bayesian Inference in Statistical Analysis, Addison-Wesley, Massachussetts.

Chen MH, Shao QM, Ibrahim JG (2000) Monte Carlo Methods in Bayesian Computation, SpringerVerlag, New York.

Coelli, T (1995) Estimators and Hypothesis Test for a Stochastic Frontier Function: A Monte Carlo Analysis, J Prod Anal 6: 247-268.

Coelli T, Henningsen A (2013) frontier: Stochastic Frontier Analysis. R package version 1.1-0. http://CRAN.R-Project.org/package=frontier (last accessed 7 October 2015).

Coelli T, Prasada Rao DS, Battese GE (1998) An Introduction to Efficiency and Productivity Analysis. Kluwer Academic Publishers, Boston.

Cuesta, R (2000) A production model with firm-specific temporal variation in technical inefficiency: With application to Spanish dairy farms. J Prod Anal 13: 139-158.

Devroye, L (1986) Non-Uniform Random Variate Generation. Springer-Verlag, New York.

Farrell MJ (1957) The measurement of productive efficiency. J Roy Stat Soc A Sta 120: 253-281.

Gelfand AE, Smith AF (1990) Sampling-based approaches to calculating marginal densities. J Am Stat Assoc 85: 398-409.

Green WH (1980) Maximun Likelihood Estimations of econometric frontier functions. J Econom 13: $27-$ 56.

Griffin JE, Steel MFJ (2007) Bayesian stochastic frontier analysis using WinBUGS. J Prod Anal 27: 163176.

Jeffreys, H (1961) Theory of Probability ( $3^{\text {nd }}$ ed). Oxford University Press, London.

Johnson N, Kotz S, Balakrishnan N (1994) Continuous Univariate Distributions (2 ${ }^{\text {nd }}$ ed). Wiley, New York.

Meeusen W, van den Broeck J (1977) Efficiency estimation from Cobb-Douglas production functions with composed error. Int Econ Rev 18: 435-444.

Olsen JA, Schmidt P, Waldman DM (1980) A Monte Carlo Study of Estimators of the Stochastic Frontier Production Function. J Econom 13: 67-82

Ortega, FJ, Basulto J, Camúñez JA (2009) Comparing Bayesian and corrected least-squares estimators in frontier production models. Boletín de Estadística e Investigación Operativa 25(2): 86-96.

Ortega FJ, Gavilan JM (2011) Algunas observaciones acerca del uso de software en la estimación del modelo half-normal. Rev Met Cuant 11: 3-16.

Pewsey A (2004) Improved likelihood based inference for the general half-normal distribution. Commun Stat-Theor M 33: 197-204.

Simar L (2007) How to Improve the Performances of DEA/FDH Estimators in the Presence of Noise?. J Prod Anal 28: 183-201

Van Den Broek J, Koop G, Osiewalski J, Steel MFJ (1994) Stochastic frontier models. A Bayesian perspective. J Econom 61: 273-303.

Whiteman J, Pearson K (1993) Benchmarking Telecommunications Using Data Envelopment Analysis. Aust Econ Pap 12: 97-105.

Zellner A (1986) On Assesing Prior Distributions and Bayesian Regression Analysis With g-Prior Distributions. Chapter 15 in Bayesian Inference and Decision Techniques, ed. By Goel P and Zellner A. Elsevier, Amsterdam. 


\section{APPENDIX: SETS OF DATA UTILIZED IN THE EXAMPLES}

Simulated sample (Example 1)

\begin{tabular}{|l|l|l|l|l|l|}
\hline $\mathbf{y}$ & 1.389195 & 0.637083 & -0.361800 & 1.365756 & 0.625770 \\
\hline $\mathbf{x}$ & 0.611111 & 0.153082 & 0.367411 & 0.593594 & 0.923927 \\
\hline
\end{tabular}

Simulated sample (Example 2)

y $\{1.08249,0.350275,0.111249,1.67043,0.804949,0.460018,1.07821,1.01255,0.141129,1.54748$, $0.296863,0.776595,1.72269,1.61751,0.957871,1.29077,0.735434,1.15074,1.22122,0.910826$, $2.25897,0.651734,0.448724,1.69737,1.04723,1.87568,1.42077,1.60624$, ।

$1.32974,1.19284,0.901887,0.453288,1.84041,0.855025,0.0904449,1.05235,1.58625,1.49994$, $0.544752,2.65495,-0.155022,1.82489,1.24477,0.711652,1.66001,0.570469,0.21666,1.00615$, $2.26923,1.40828\}$

$\mathbf{X}_{2}\{0.728113,0.50603,0.212999,0.755097,0.684416,0.367649,0.0441774,0.934338,0.340176$, $0.572793,0.350698,0.893679,0.195965,0.876396,0.80044,0.680444,0.0483495,0.196176$, $0.350175,0.553006,0.671351,0.20208,0.10168,0.399454,0.490328,0.844086,0.529784$, $0.871228,0.162341,0.851186,0.699436,0.15938,0.453054,0.218776,0.211959,0.514752$, $0.675255,0.659948,0.872841,0.879089,0.319521,0.891303,0.191057,0.955342,0.0475877$, $0.149271,0.117269,0.822466,0.83501,0.201067\}$

$\mathbf{X}_{3}\{0.620443,0.797728,0.486157,0.254354,0.909706,0.65939,0.174122,0.885507,0.114798$, $0.607201,0.708227,0.0685596,0.613377,0.802765,0.40258,0.0449246,0.598802,0.144266$, $0.193644,0.649677,0.783038,0.0725557,0.662766,0.4495,0.202687,0.614011,0.990142,1$

$0.370271,0.771085,0.0345378,0.609937,0.186131,0.444118,0.725772,0.896521,0.309074$, $0.573408,0.370633,0.792472,0.798635,0.280654,0.922334,0.080111,0.21696,0.834997$, $0.311379,0.167264,0.93436,0.626333,0.446917\}$

Set of data on the production and inputs in the example of telecommunications (Section 5).

\begin{tabular}{|c|c|c|c|}
\hline Country & Output Index & Main lines & $\begin{array}{c}\text { Number of } \\
\text { Employees } \\
\left(10^{6} \mathrm{~km}\right)\end{array}$ \\
\hline Australia & 0.74 & 0.7767 & 0.85 \\
\hline Austria & 0.24 & 0.3223 & 0.18 \\
\hline Belgium & 0.36 & 0.399 & 0.26 \\
\hline Canada & 1.26 & 1.5296 & 1.05 \\
\hline Denmark & 0.39 & 0.2911 & 0.18 \\
\hline Finland & 0.29 & 0.267 & 0.2 \\
\hline France & 2.06 & 2.8085 & 1.56 \\
\hline Germany & 1.73 & 2.9981 & 2.12 \\
\hline Iceland & 0.02 & 0.0126 & 0.02 \\
\hline Ireland & 0.11 & 0.0983 & 0.13 \\
\hline Italy & 1.48 & 2.2350 & 1.18 \\
\hline Japan & 2.73 & 5.3236 & 2.77 \\
\hline The & 0.77 & 0.694 & 0.32 \\
\hline Netherlands & 0.16 & 0.1473 & 0.17 \\
\hline Zealand & 0.27 & 0.2132 & 0.15 \\
\hline Norway & 0.19 & 0.2379 & 0.23 \\
\hline Portugal & & & \\
\hline
\end{tabular}




\begin{tabular}{|c|c|c|c|} 
Spain & 0.59 & 1.2603 & 0.75 \\
\hline Sweden & 0.71 & 0.5849 & 0.42 \\
\hline Switzerland & 0.56 & 0.3943 & 0.22 \\
\hline Turkey & 0.15 & 0.6893 & 0.36 \\
\hline UK & 2.53 & 2.5404 & 2.27 \\
\hline
\end{tabular}

